\title{
Functioning in individuals with chronic fatigue syndrome: increased impairment with co-occurring multiple chemical sensitivity and fibromyalgia
}

\author{
Molly M Brown*† and Leonard A Jason ${ }^{\dagger}$
}

Address: Department of Psychology, DePaul University, Center for Community Research, Chicago, IL, USA

Email: Molly M Brown* - mbrown59@depaul.edu; Leonard A Jason - ljason@depaul.edu

* Corresponding author †Equal contributors

Published: 30 July 2007

Dynamic Medicine 2007, 6:9 doi:10.1 186/1476-5918-6-9

This article is available from: http://www.dynamic-med.com/content/6/I/9

(c) 2007 Brown and Jason; licensee BioMed Central Ltd.

This is an Open Access article distributed under the terms of the Creative Commons Attribution License (http://creativecommons.org/licenses/by/2.0), which permits unrestricted use, distribution, and reproduction in any medium, provided the original work is properly cited.

\section{Correction}

In our publication [1], the frequencies and percentages of lifetime and current Axis I disorders reported in Table 1 were found to be inaccurate. The values reported in Table 1 of this version have been corrected. All other outcome variables in Table 1 were reported correctly in the original version of the article. The analyses and results in the text were accurate in the original version of the paper.

\section{References}

I. Brown MM, Jason LA: Functioning in individuals with chronic fatigue syndrome: increased impairment with co-occurring multiple chemical sensitivity and fibromyalgia. Dynamic Medicine 2007, 6:6
Received: 18 June 2007

Accepted: 30 July 2007 
Table I: Outcome measures for individuals with CFS alone; CFS-MCS; CFS-FM; or CFS-MCS-FM

\begin{tabular}{|c|c|c|c|c|}
\hline Outcome Measure & CFS alone $(n=50)$ & CFS-MCS $(n=27)$ & CFS-FM $(n=18)$ & CFS-MCS-FM $(n=19)$ \\
\hline \multicolumn{5}{|l|}{ Mean (Standard Deviation) } \\
\hline \multicolumn{5}{|l|}{ MOS-SF-36 } \\
\hline Physical Functioning & $53.6 \mathrm{I}(25.05)^{\mathrm{a}}$ & $42.50(19.66)$ & $40.56(23.69)$ & $32.89(19.95)^{\mathrm{a}}$ \\
\hline Role-Physical & 4.69 (12.27) & $3.85(11.60)$ & $4.17(12.86)^{\prime}$ & $3.95(12.54)$ \\
\hline Bodily Pain & $49.83(21.88)^{\mathrm{a}, \mathrm{c}}$ & $40.62(2 \mathrm{I} .63)^{\mathrm{b}}$ & $30.00(13.18)^{c}$ & $21.58(18.60)^{a, b}$ \\
\hline General Health & $36.65(16.74)^{\mathrm{a}}$ & $28.75(15.94)$ & $38.54(16.62)^{b}$ & $19.95(32.24)^{a, b}$ \\
\hline Vitality & $20.73(15.98)$ & $15.19(12.84)$ & $19.72(16.31)$ & $11.32(9.84)$ \\
\hline Social Functioning & $46.09(25.15)^{\mathrm{a}}$ & $30.29(21.84)^{a}$ & $47.22(23.70)$ & $30.26(19.68)$ \\
\hline Role-Emotional & $56.74(45.00)$ & 47.44 (45.39) & $42.59(35.80)$ & $57.89(41.34)$ \\
\hline Mental Health & $67.08(16.30)$ & $55.85(19.35)$ & 66.22 (14.79) & $62.11(18.10)$ \\
\hline \multicolumn{5}{|l|}{ Brief COPE } \\
\hline Self Distraction & $2.30(0.87)^{\mathrm{a}, \mathrm{b}}$ & $2.9 \mathrm{I}(0.8 \mathrm{I})^{\mathrm{b}}$ & $2.89(0.88)$ & $3.18(0.65)^{\mathrm{a}}$ \\
\hline Active Coping & $2.97(0.84)$ & $3.15(0.83)$ & $3.28(0.73)$ & $3.10(0.88)$ \\
\hline Denial & $1.41(0.85)$ & $1.28(0.59)$ & $1.25(0.35)$ & $1.32(0.61)$ \\
\hline Substance Use & $1.15(0.47)$ & $1.22(0.63)$ & $1.53(0.98)$ & $1.24(0.42)$ \\
\hline Emotional Support & $2.40(0.95)$ & $2.17(0.91)$ & $2.56(0.97)$ & $2.29(0.85)$ \\
\hline Instrumental Support & $2.32(0.96)$ & $2.61(0.93)$ & $2.78(0.75)$ & $2.66(0.99)$ \\
\hline Behavioral Disengagement & $\mathrm{I} .33(0.5 \mathrm{I})$ & $1.27(0.38)$ & $\mathrm{I} .47(0.44)$ & $\mathrm{I} .47(0.94)$ \\
\hline Venting & $1.93(0.63)$ & $2.09(0.77)$ & $2.39(0.65)$ & $2.11(.077)$ \\
\hline Positive Reframing & $2.20(1.06)$ & $2.28(0.94)$ & $2.75(0.96)$ & $2.53(1.05)$ \\
\hline Planning & $2.97(0.97)$ & $3.00(0.98)$ & $3.00(0.86)$ & $3.03(0.86)$ \\
\hline Humor & $1.68(0.79)^{\mathrm{a}}$ & $1.78(0.87)$ & $2.39(1.20)^{\mathrm{a}}$ & $1.79(0.77)$ \\
\hline Acceptance & $2.95(0.88)$ & $2.81(0.83)$ & $2.94(0.75)$ & $2.95(0.80)$ \\
\hline Religion & $2.4 I(1.01)$ & $2.44(0.97)$ & $2.31(1.06)$ & $2.47(1.05)$ \\
\hline Self Blame & $1.69(0.87)$ & $1.74(0.75)$ & $2.00(0.77)$ & $1.92(0.79)$ \\
\hline Beck Depression Inventory & I $6.47(8.61)^{\mathrm{a}}$ & $20.88(9.64)$ & $18.50(9.54)$ & $23.93(11.43)^{a}$ \\
\hline Fatigue Severity Scale & $5.98(0.68)$ & $6.35(0.66)^{a}$ & $5.70(1.22)^{\mathrm{a}}$ & $6.30(0.53)$ \\
\hline Pittsburgh Sleep Quality Index & $7.26(2.53)^{\mathrm{a}}$ & $8.69(2.20)$ & $8.94(2.10)$ & $8.95(2.39)^{a}$ \\
\hline \multicolumn{5}{|l|}{ Brief Pain Inventory } \\
\hline Interference & $2.83(2.52)^{\mathrm{a}, \mathrm{c}, \mathrm{d}}$ & $4.56(2.78)^{b, c}$ & $5.36(1.96)^{d}$ & $6.80(2.33)^{a, b}$ \\
\hline Severity & $3.03(2.06)^{\mathrm{a}, \mathrm{c}}$ & $4.06(2.02)^{b}$ & $4.86(1.40)^{c}$ & $6.10(2.04)^{\mathrm{a}, \mathrm{b}}$ \\
\hline \multicolumn{5}{|l|}{ Symptoms } \\
\hline Sore Throat & $23.50(26.61)$ & $29.48(26.97)$ & $20.56(26.40)$ & $24.84(28.82)$ \\
\hline Tender Lymph Nodes & $25.75(30.36)$ & $23.40(26.33)$ & $27.50(33.79)$ & $32.00(27.92)$ \\
\hline Muscle Pain & $50.52(28.34)^{a, b}$ & $60.76(28.22)$ & $72.92(22.56)^{b}$ & $78.42(24.97)^{\mathrm{a}}$ \\
\hline Joint Pain & $31.85(33.25)^{\mathrm{a}, \mathrm{c}}$ & $46.04(35.95)^{b}$ & $63.06(32.18)^{c}$ & $75.50(24.54)^{a, b}$ \\
\hline Impaired Memory & $62.96(25.22)$ & $59.63(23.82)$ & $65.14(25.53)$ & $68.94(22.65)$ \\
\hline Unrefreshing Sleep & $76.89(18.89)$ & $75.19(25.43)$ & $89.67(10.86)$ & $87.03(14.46)$ \\
\hline Post-Exertional Malaise & $73.27(17.84)$ & $75.19(19.16)$ & $74.86(23.74)$ & $82.36(14.10)$ \\
\hline Headaches & $42.91(33.39)^{\mathrm{a}}$ & $56.87(30.50)$ & $55.00(31.44)$ & $74.03(24.39)^{\mathrm{a}}$ \\
\hline \multicolumn{5}{|l|}{ Six Minute Walk Test } \\
\hline Distance Walked (feet) & $1419.47(312.17)$ & $1728.08(2387.62)$ & | 304.28 (356.492) & $1221.39(239.23)$ \\
\hline Average RPE & $9.85(2.24)^{\mathrm{a}}$ & $10.56(2.08)$ & $11.37(2.76)$ & $11.78(2.42)^{\mathrm{a}}$ \\
\hline Sit and Reach (inches) & $13.48(3.54)$ & $13.16(4.49)$ & $14.55(4.24)$ & $13.40(3.77)$ \\
\hline \multicolumn{5}{|l|}{ Hand Grip (pounds) } \\
\hline Right & $64.45(24.67)$ & $61.57(21.21)$ & $64.19(21.87)$ & $52.28(15.34)$ \\
\hline Left & $60.04(22.71)$ & $58.63(17.90)$ & $58.72(20.7 \mathrm{I})$ & $48.07(17.21)$ \\
\hline Actigraphy Mean & $161.35(58.55)$ & 145.74 (58.13) & $167.23(48.51)$ & $149.78(63.12)$ \\
\hline Lifetime Axis I Diagnosis \% & $29(58.0 \%)$ & $17(63.0 \%)$ & $12(66.7 \%)$ & $13(68.4 \%)$ \\
\hline Current Axis I Diagnosis \% & $19(38.0 \%)$ & $8(29.6 \%)$ & $9(50.0 \%)$ & $8(42.1 \%)$ \\
\hline
\end{tabular}

Similar letters across diagnostic groups significantly differ at the $p<.05$ level. 